\title{
L'aggiornamento dei docenti di italiano L2. Una ricerca sulle necessità formative degli insegnanti
}

\author{
Professional Training and Updating of Italian as Second \\ Language Teachers. A Research on Teachers' Training Needs
}

\author{
Giulio Facchetti \\ Università degli Studi dell'Insubria, Italia \\ Micaela Grosso \\ Università ECampus, Italia
}

Paolo Nitti ${ }^{1}$

Università degli Studi dell'Insubria, Italia

\begin{abstract}
Riassunto: In questo contributo sono discussi i risultati di un questionario relativo alle necessità formative del personale docente, che si occupa di insegnamento dell'italiano L2. A partire dalla valutazione della letteratura scientifica di riferimento, è stato strutturato un questionario per rilevare i bisogni formativi e gli aspetti critici in merito alla dimensione della didattica dell'italiano L2. Dall'analisi dei dati emerge con chiarezza il desiderio di formazione di carattere linguistico e la necessità di ulteriori raccordi fra mondo accademico e professionale.

Parole chiave: aggiornamento professionale, didattica L2, lingua italiana, glottodidattica, linguistica italiana, linguistica educativa.

Abstract: In this essay, we discuss the results of a questionnaire to observe the perception of the needs associated with the professional training of Italian as Second Language teachers. Previously, we investigated these necessities according to a scientific literature review. A questionnaire was administered to a sample of teachers in order to explore the professional needs and the critical aspects of Italian as Second Language Teaching. From data analysis two significant needs emerge. The first being the formal instruction of language training, and the second being to improve the communication between academic research and professional life.
\end{abstract}

Keywords: professional training, SL teaching, Italian language, language teaching, Italian Linguistics, Educational Linguistics.

\footnotetext{
I Sebbene il contributo sia concepito in forma unitaria, a Giulio Facchetti sono attribuiti i paragrafi 4.2 e
} 5.2, a Micaela Grosso i paragrafi 2, 3, 4.1, le conclusioni e a Paolo Nitti l'introduzione e i paragrafi 1 e 5.1. 


\section{Introduzione}

L'aggiornamento del personale docente costituisce, a prescindere dal periodo storico, dal contesto socio-culturale e dalla disciplina insegnata, un fattore imprescindibile dei processi educativi. Qualsiasi forma di insegnamento, infatti, con il passare del tempo tende a perdere il proprio collegamento con la realtà e diviene anacronistica (Emanuel, Nitti 2017). Si ricorda, in effetti, che gli approcci che si sono susseguiti, per quanto concerne l'insegnamento delle lingue, riflettevano - e tuttora delineano - una "filosofia di fondo: I'idea che si ha di lingua, di cultura, di comunicazione, di studente, di insegnante, di insegnamento" (Balboni, 2012: 6), e che tale idea riflette fattori di carattere storico-culturale.

Secondo il rapporto OCSE TALIS 2013, inoltre, la partecipazione degli insegnanti in Italia alle attività di aggiornamento professionale risulta una tra le più basse in merito ai Paesi partecipanti all'indagine (75\% Italia, $88 \%$ media TALIS), evidenziando un calo del $10 \%$ rispetto al 2008. II numero di docenti che dichiara di aver beneficiato di forme di incentivi, per lo più di carattere economico, rispetto alle attività di aggiornamento è ancora più sottile e occorrerebbe aggiungere il corpo docente che non è impiegato nei cicli tradizionali di istruzione e che lavora in contesti privati.

Ultimamente il Ministero dell'Università e della Ricerca riconosce agli insegnanti di ruolo che operano nei cicli di istruzione ordinari (scuola dell'infanzia, primaria e secondaria) ${ }^{2}$ un buono spendibile in attività formative e in altri tipi di benefit.

Sulla base di queste premesse è parso opportuno investigare i bisogni formativi percepiti dagli insegnanti di italiano L2, attraverso l'identificazione di un campione rappresentativo, considerando che la letteratura scientifica è ricca di contributi di carattere speculativo, ma sostanzialmente povera in termini di ricerca di carattere applicativo.

Si denuncia, in substantia, una parcellizzazione significativa delle trattazioni delle indagini connesse con i bisogni formativi degli insegnanti, a discapito di una visione unitaria (Nitti, 2018). Nessuna rivista, inoltre, si occupa in maniera specifica della formazione degli insegnanti di lingua, mentre il panorama italiano è ben nutrito di sedi che trattano la didattica delle lingue e, in particolare, dell'italiano L2.

I bisogni formativi degli insegnanti dipendono da molti fattori e, primariamente, dal contesto territoriale all'interno del quale i docenti operano. La ricerca, pertanto, è circoscritta al Comune di Torino, sebbene possa offrire un quadro rappresentativo di molte realtà territoriali in Italia e all'estero.

\section{I bisogni formativi}

Le necessità formative degli insegnanti possono essere suddivise a seconda di un'attenzione:

1) All'amministrazione e all'agenzia formativa;

\footnotetext{
2 "ai docenti di ruolo a tempo indeterminato delle Istituzioni scolastiche statali, sia a tempo pieno che a tempo parziale, compresi i docenti che sono in periodo di formazione e prova, i docenti dichiarati inidonei per motivi di salute di cui all'art. 514 del Dlgs. 16/04/94, n.297, e successive modificazioni, i docenti in posizione di comando, distacco, fuori ruolo o altrimenti utilizzati, i docenti nelle scuole all'estero, delle scuole militari" (https://cartadeldocente.istruzione.it/\#/ - ultima consultazione 21/06/2019).
} 
2) Alla qualità della didattica;

3) Alla gestione degli studenti come singoli e come gruppi;

4) Al contenuto della disciplina in termini di aggiornamento.

Nel primo caso si tratta essenzialmente dei rapporti fra l'insegnante e l'istituzione presso la quale opera: la formazione, pertanto, si rivolge alle capacità di attrarre fondi e interesse per attività didattiche, ai corsi relativi alla sicurezza e alle necessità contingenti rispetto alla struttura e al territorio.

Per quanto concerne, invece, la qualità della didattica, i bisogni formativi sono decisamente più complessi e riguardano essenzialmente la gestione del tempo, le modalità didattiche intese come strategie e tecniche, la capacità di utilizzo di software e hardware ad uso didattico (glottotecnologie).

La gestione degli apprendenti è un'altra necessità formativa particolarmente rilevante. Occorre menzionare, nella fattispecie, la didattica rivolta a contesti disomogenei (Nitti, 2018), la presenza di studenti caratterizzati da bisogni educativi speciali, l'utilizzo di formati didattici ludici, la didattica cooperativa e collaborativa (Dörnyei, 1998).

L'ultimo caso riguarda un punto di intersezione fra l'istituzione e gli studenti, poiché la didattica collaborativa si riferisce alla capacità di gestire una lezione, un'unità didattica, un programma e, più in generale, qualsiasi processo didattico con i propri colleghi.

II contenuto disciplinare, invece, costituisce uno dei bisogni sicuramente meno percepiti, soprattutto considerati gli anni necessari alla formazione per divenire insegnanti, tuttavia si rivela indispensabile, perché riguarda l'oggetto dell'azione didattica (Peacock, 1997).

La ricerca accademica, infatti, progredisce nel corso del tempo e si assiste a un avanzamento degli studi per quanto concerne le discipline insegnate; un esempio ben circostanziato riguarda la dicotomia "direzionalità/bimodalità" (Danesi, 2015). Secondo gli studiosi della seconda metà del Novecento, il cervello percepisce e apprende una lingua attivando entrambi gli emisferi cerebrali, ma dando la precedenza a quello destro, in modo che il materiale linguistico sia percepito globalmente e, solo in seguito, scomposto, analizzato e processato.

In effetti, gli studi più recenti hanno evidenziato come gli emisferi interagiscano fin dai primi momenti della percezione del materiale linguistico, rendendo molto più elastico il fattore della direzionalità (Marini, 2018).

I docenti di lingua che si sono formati prima che la dicotomia fosse posta in discussione hanno imparato che la didattica della lingua deve osservare necessariamente una dimensione globale, alla quale seguirà una più analitica. Così, senza un aggiornamento di carattere glottodidattico, non sarà possibile proporre interventi scientifici attuali e si produrranno forme di apprendimento certamente meno valide sul piano scientifico e qualitativo. Non si intende affermare, ovviamente, che un insegnante poco aggiornato produca necessariamente apprendimenti inefficaci, ma si tratta di operare a seconda di una prospettiva scientifica, in quanto la didattica acquisizionale ha per obiettivo di modellizzare "la relazione tra un certo 
tipo di insegnamento e un certo tipo (velocità, ritmo, qualità) di apprendimento" (Rastelli, 2009: 25).

Non sempre, infine, i bisogni formativi che i docenti percepiscono aderiscono perfettamente alle sfide della contemporaneità e ai risultati del progresso scientifico; in molti casi si colgono dei tentativi di migliorare la qualità dell'insegnamento delle lingue straniere (Mezzadri, 2005) o, più semplicemente, si è mossi dalla curiosità o dagli obblighi di aggiornamento professionale.

\section{L'aggiornamento del personale docente e il lifelong learning}

Come si è visto, con l'evoluzione della società della conoscenza, sono emerse nuove esigenze di formazione, che si sono affiancate a delle tendenze evolutive di cui le future politiche d'innovazione dovranno necessariamente tenere conto. Infatti, il contesto che si è venuto a creare negli ultimi anni costringe, in senso stretto, alla presa di posizione in direzione di una diversificazione dell'intervento formativo. Grazie ai progressi compiuti dalla scienza, dalla medicina e dalla tecnologia, si assiste oggigiorno a un allungamento dell'aspettativa di vita e questa è una condizione che impone il dovere di rivolgere particolare attenzione alle prime fasi dello sviluppo umano, ma al contempo impedisce di ignorare le successive e le ultime, altrettanto importanti (Frabboni, Pinto Minerva, 2005).

La tendenza contemporanea, a livello politico e socio-economico, è quella di un impulso all'apprendimento su larga scala, diffuso e promosso in diversi contesti di vita e a vantaggio di differenti profili di apprendente. In quest'ottica, sarebbe bene considerare il lifelong learning come nuova identità (Emanuel, Nitti, 2017), organica e coerente della formazione, anziché come intervento articolato in azioni formative distinte.

Per Frabboni e Pinto Minerva (2005), ciò condurrebbe a una condizione nella quale, affiancato alla richiesta sempre crescente di formazione ad hoc, si accompagnerebbe il divario emergente tra classi sociali distinte da un libero accesso alla cultura e classi sociali meno provviste di risorse, culturali o economiche. Il compito delle istituzioni, in questo senso, dovrebbe appunto essere quello di lavorare in direzione di un appianamento del dislivello, attraverso la promozione della formazione permanente e rivolta a tutti, in modo da poter sopperire ai bisogni personali, sociali, lavorativi dei cittadini.

In questo quadro, gli insegnanti si sono spesso trovati a dover far fronte a all'inserimento di studenti stranieri nelle proprie classi, e all'obbligo, dunque, di un cambio prospettico al fine di sostenere bisogni formativi variati.

Nel 2006, il MIUR ha pubblicato delle Linee guida per l'accoglienza e l'integrazione degli alunni stranieri, riviste poi nel 2014; nel documento ${ }^{3}$ sono presentate alcune buone pratiche utili alla gestione della classe e alla promozione dei gruppi plurilingue. Al docente, secondo le Linee, sono richieste diverse competenze specifiche, tra le quali vi è la semplificazione della lingua utilizzata in aula, una valida iniziale comunicazione non verbale, la capacità d'esplicitazione delle consegne e dei testi delle diverse discipline. Inoltre, è richiesta ai docenti una competenza nelle TIC

3 Cfr. http://www.istruzione.it/archivio/web/ministero/focus190214.html (ultima consultazione 23/06/2019). 
(Tecnologie dell'Informazione e della Comunicazione), che possa creare coinvolgimento $e$ interesse sia tra gli studenti italofoni che tra quelli stranieri (Santalucia, 2015). Un'ulteriore abilità richiesta è quella relativa all'assetto docimologico (Serragiotto, 2016), in itinere e sommativa, delle competenze degli studenti, nonché l'adozione di un approccio interculturale alla trattazione delle materie di studio, che favorisca l'integrazione ed eviti per quanto possibile la genesi di conflitti (Dusi, Guidetti, Portera, 2015).

Da quanto indicato emerge un urgente bisogno di formazione preliminare e nel corso della carriera, sia nei contesti tradizionali di scuola dell'obbligo, ma ancor di più in ambienti non regolamentati dalle direttive del MIUR quali enti privati di formazione, centri interculturali e il mondo variegato dell'associazionismo e del privato-sociale.

\section{La classe A-23}

Dalle indicazioni fornite nelle Linee guida per l'accoglienza e l'integrazione degli alunni stranieri, emerge un profilo definito del docente esperto nell'insegnamento della L2, che combina le competenze del docente di italiano e di quello di lingua straniera, e le integra con un bagaglio glottodidattico specialistico.

Soltanto da qualche tempo, però, il profilo dell'insegnante di L2 ha acquisito in Italia una propria autonomia, grazie all'istituzione della classe di concorso A23, sebbene il nuovo quadro non si possa dire risolutivo. L'accesso al concorso è stato infatti possibile ai docenti abilitati all'esercizio della professione, ma, com'è noto, non esiste un'abilitazione specifica per la didattica dell'italiano per stranieri. Inoltre, molti insegnanti in possesso di anni di esperienza non hanno potuto assistere al riconoscimento del proprio bagaglio perché non in possesso dei CFU-ECTS riconosciuti come requisiti per l'accesso quali, ad esempio, la lingua e letteratura latine.

Dei percorsi universitari post lauream (specializzazioni e master italiani di primo e di secondo livello) e dei corsi di specializzazione riconosciuti dal Ministero, poi, vi è da dire che il tratto accomunante in molti casi è la richiesta, per l'ammissione, di lauree di provenienza che, inspiegabilmente, non collimano con l'elenco dei percorsi segnalati per l'accesso alla A-23.

Di qui l'obbligo non solo di una maggiore e più fine delineazione della figura dell'insegnante di italiano L2, ma anche della creazione di percorsi abilitanti progettati al fine di portare gli aspiranti alla cattedra a un livello superiore di competenza glottodidattica, uniformemente e coerentemente.

\section{La ricerca e il campione}

\subsection{La ricerca}

Alla luce delle osservazioni esposte nei paragrafi precedenti, l'obiettivo della ricerca è stato individuato nell'intenzione di inquadrare i bisogni formativi specifici degli insegnanti di italiano come L2, con il fine di tracciarne un profilo aggiornato e utile all'elaborazione di una strategia formativa efficace, poiché adeguata e conforme alle aspettative. 
Per il reperimento dei rispondenti, ci si è mossi attraverso una mailing list precedentemente elaborata e composta da un elenco di nominativi consolidati4. II primo contatto ha previsto l'invio, alle persone presenti nella banca dati, di un messaggio di posta elettronica con una domanda relativa all'intenzione o meno di partecipare alla ricerca, i cui obiettivi erano esplicitati nel testo dell'e-mail. Dalle risposte positive, è stato ricavato il campione dei rispondenti, composto da 147 docenti di italiano L2, su un totale di 198 individui contattati. In seguito, è stato proposto un questionario strutturato su Google Moduli, composto da 30 quesiti a risposta chiusa e 5 a risposta aperta, per un totale di 35.

II questionario sottoposto agli informanti era ripartito in tre sezioni, ciascuna afferente a un'area d'indagine specifica. La prima sezione, d'inquadramento del campione, riportava 8 domande di carattere socio-anagrafico quali l'età, il genere, il titolo di studio, gli anni di esperienza, il comune di residenza, il contesto lavorativo e il tipo di istituto d'appartenenza, le eventuali specializzazioni in didattica della L2 e delle lingue, più in generale.

II secondo nucleo di domande era costituito da 11 quesiti più squisitamente tecnici e glottodidattici, con richieste relative alle abilità linguistiche coinvolte nella scansione delle unità di acquisizione, alle tecniche di gestione della classe, alle strategie adottate, alle riflessioni sull'insegnamento del lessico e della grammatica, alle criticità dell'intervento in contesto plurilingue, alla gestione e correzione degli errori, alle tempistiche adottate e ai materiali adottati.

Nell'ultima sezione, la più lunga, poiché composta da 16 quesiti, si richiedeva di entrare nel merito della propria necessità formativa e delle competenze didattiche, organizzative e professionali desiderate. Gli ultimi quesiti, indagavano più ampiamente i bisogni sulla base della posizione ricoperta, della ricezione delle Linee Guida e della classe di concorso A-23, delle caratteristiche dei propri studenti, con particolare attenzione alle criticità riscontrate e alle possibili risoluzioni a breve, medio e lungo termine.

\subsection{II campione}

\footnotetext{
${ }^{4}$ Si tratta di docenti che hanno seguito percorsi di formazione in didattica dell'italiano L2 al Centro Interculturale della Città di Torino (www.intercultura.torino.it - ultima consultazione 23/06/2019).
} 
La maggior parte degli informanti, come emerge dal Grafico 1, lavora all'interno della scuola dell'obbligo, mentre una parte decisamente più ridotta opera all'interno dell' associazionismo

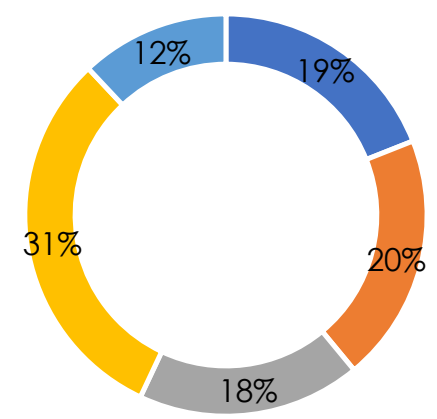

- Primaria

- Secondaria primo grado

- Secondaria secondo grado

- CPIA - Istruzione adulti

- Associazioni e volontariato

Grafico 1 - Le agenzie formative del campione

Per quanto concerne il titolo di studio, come si nota nel Grafico 2, la maggioranza è in possesso di una laurea di primo livello e si nota una componente significativa del campione in possesso di certificazioni di carattere glottodidattico.

Il possesso del solo diploma o maturità di scuola secondaria di secondo grado riguarda essenzialmente i docenti che operano all'interno della scuola primaria o dell'associazionismo.

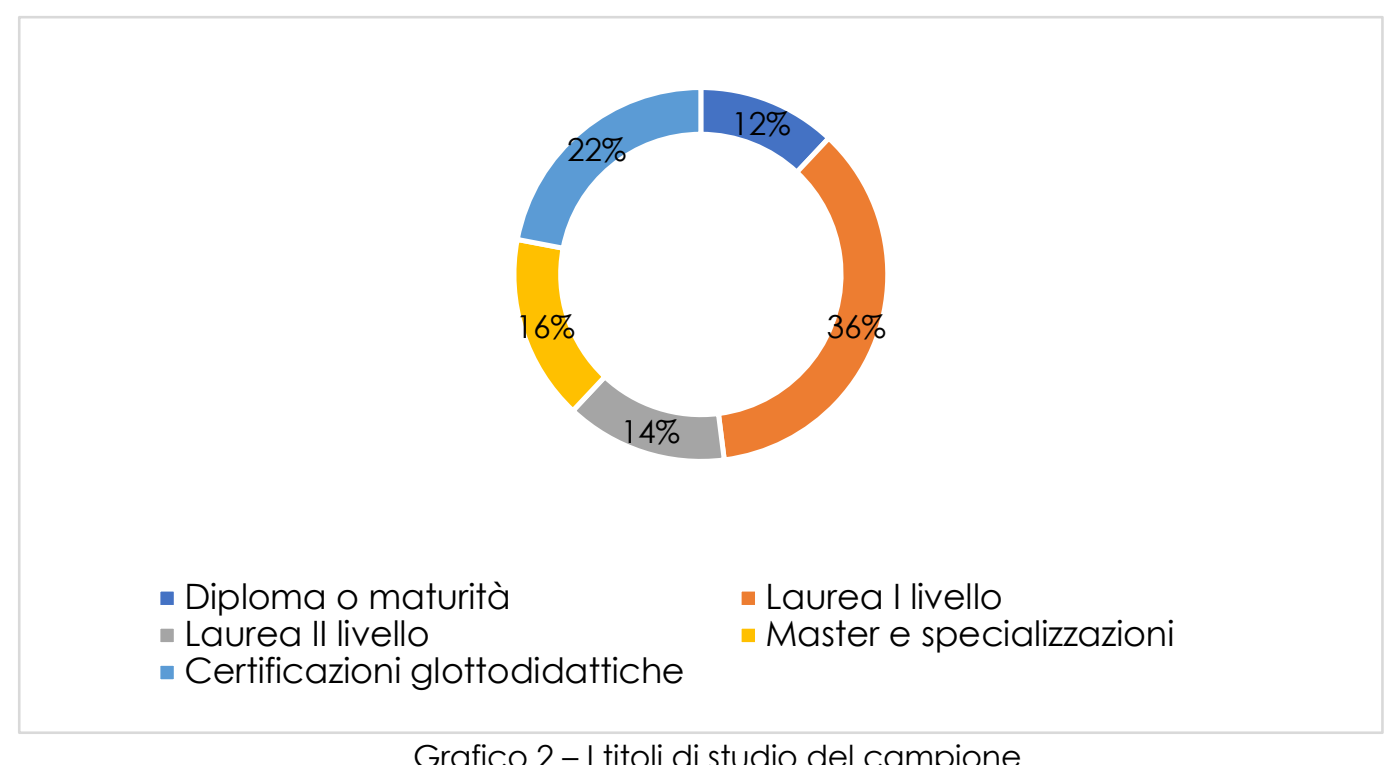

Grafico 2 - I titoli di studio del campione 


\section{Analisi dei dati}

\subsection{I bisogni formativi come sfide per la glottodidattica}

Come si è visto, sulla base delle premesse alla ricerca, individuate nel $\S 3$, è stato strutturato un questionario di carattere qualitativo, rivolto a un campione di 147 docenti di italiano L2. II contesto di lavoro degli informanti che hanno partecipato alla ricerca risulta certamente significativo in merito alle risposte e agli aspetti su cui investire: molti (47\%) lamentano, infatti, di lavorare in contesti troppo piccoli e limitanti, chiedendo, di conseguenza, un suggerimento di modalità didattiche efficaci laddove sia problematico far lavorare i propri studenti in gruppo.

Il dato maggiormente significativo, in effetti, è quello legato alla condizione di disomogeneità dei gruppi classe (98\%). Si tratta di contesti non necessariamente numerosi rispetto alla quantità di corsisti, ma decisamente eterogenei per quanto concerne la dimensione della scolarizzazione, l'età, il livello linguistico e lingue di provenienza (Nitti, 2018). La gestione di queste classi può essere problematica, specie se non si considera la didattica ad abilità differenziate e la gestione dei nuclei fondanti rispetto ai sillabi e ai curricoli.

Nelle situazioni più estreme, alcuni docenti (8\%) lamentano la presenza di corsisti analfabeti e alfabetizzati all'interno dello stesso gruppo classe. In questi casi occorrerebbe certamente, più che aggiornare e formare il corpo docente, rimodulare l'organizzazione dell'agenzia formativa. All'interno di pratiche formative, tuttavia, è possibile suggerire modalità di lavoro ad abilità differenziate, consigliando anche di lavorare di più sull'oralità che accomuna l'intero gruppo. Quest'ultima considerazione risulta particolarmente efficace, se si pensa che quasi tutte le lingue naturali siano prioritariamente parlate e, solo secondariamente ed eventualmente, scritte (Facchetti, 2007).

Un altro bisogno formativo significativo, evidenziato da tutti gli insegnanti che lavorano all'interno delle scuole primarie e secondarie (65\% del campione), riguarda la necessità di aggiornarsi rispetto alle strategie per veicolare e sviluppare la lingua dello studio.

L'italiano per lo studio, difatti, costituisce un sottocodice a tutti gli effetti, delineandosi come settore della lingua specializzato, contraddistinto da una forte componente primariamente di carattere diastratico e secondariamente diafasico (Berruto, 2004).

Uno dei bisogni formativi percepiti da una netta minoranza del campione $134 \%$ di cui $97 \%$ insegnanti di scuola secondaria di primo e di secondo grado) concerne le procedure e le modalità di valutazione degli apprendenti, sia per quanto concerne l'asse certificatorio che il piano autovalutativo (Serragiotto, 2016). L'autovalutazione degli insegnanti, invece, non è stata segnalata come risposta da nessun informante e risulta un dato significativo, perché permette di identificare una tendenza.

\subsection{L'aggiornamento nella disciplina linguistica}

Il desiderio di aggiornarsi sul contenuto disciplinare - nel nostro caso la lingua italiana - risulta evidente per la maggioranza del campione (88\%) e il dato non sorprende, poiché "per quanto concerne l'aggiornamento linguistico, è fondamentale che i docenti restino in contatto con le lingue straniere insegnate o continuino a 
confrontarsi con la propria lingua, nel caso dell'insegnamento della lingua seconda" (Emanuel, Nitti, 2017: 24). II confronto costante con la lingua che si insegna consente di essere preparati sul piano testuale e su quello sociolinguistico, offrendo ai propri discenti i testi e le varietà della lingua effettivamente utilizzate; si evita in questo modo la proposta in classe di modelli desueti e superati.

I dati sulle tecniche e sull'insegnamento del lessico e della grammatica mostrano come la lezione frontale rappresenti il formato didattico (Balboni, 2012) ancora privilegiato (64\% delle risposte) e la visione della grammatica come insieme prescrittivo di regole (78\% delle risposte) prevale rispetto a una prospettiva sistemica (23\% delle scelte).

In aggiunta a quanto riferito, il desiderio di aggiornarsi sulle pratiche di insegnamento e sugli stili di apprendimento rappresenta un altro aspetto particolarmente evidente dell'analisi dei dati (67\%): in molti casi, infatti, si è quasi alla ricerca di una sorta di ricetta miracolosa che risolva i problemi all'interno del contesto d'aula. In questi casi, mentre chi insegna è rivolto al prodotto dell'insegnamento, sul piano della ricerca non ci si "limita ai prodotti linguistici, ma si cerca di osservare i processi che li generano" (Rastelli in Nuzzo, Rastelli, 2011 : 17).

Plausibilmente, la dicotomia individuata da Rastelli fra prodotto e processo rappresenta proprio una delle caratteristiche della percezione dei bisogni formativi da parte dei docenti: il mondo accademico è interessato ai processi, mentre il personale docente è orientato alla piena applicabilità, ai prodotti e bisognerebbe certamente individuare degli orizzonti intermedi.

\section{Conclusioni}

Dai risultati ottenuti dal questionario, è possibile evincere una forte necessità formativa, estesa al personale docente e trasversale ai livelli e ai contesti di insegnamento.

Contemporaneamente, l'occasione fornisce più spunti di riflessione sulla formazione, pertanto, non solo di chi svolge attualmente la professione di insegnante di italiano L2, ma anche dei futuri abilitati. Tematiche quali le buone pratiche e le criticità dell'insegnamento della $L 2$, la competenza interculturale, le tecniche di sviluppo delle abilità integrate, lo sviluppo della lingua dello studio, le tempistiche di acquisizione dell'italiano di base e della lingua dello studio (Favaro, 2014), risultano tasselli necessari da acquisire per portare a termine il compito del docente.

Particolare importanza assume l'assenza di risposte relative all'autovalutazione da parte dei docenti che, come si diceva, segnala una tendenza. Che si tratti di una tematica sottovalutata o che l'autovalutazione venga recepita come passaggio non cruciale nell'inquadramento didattico quotidiano, il dato comunica una percezione manchevole dei rispondenti.

II rapporto tra Scuola e Università, poi, è un requisito essenziale per la buona riuscita di un qualsivoglia intervento di formazione o aggiornamento; gli atenei, cui andrebbe il compito dell'individuazione, attraverso una rigorosa ricerca scientifica, di estremi teorici e metodi utili alla trattazione delle attuali urgenze e lacune operative della Scuola, dovrebbero con quest'ultima poter dialogare per effettuare un'analisi dei bisogni approfondita e mirata. 
In Italia, purtroppo, nel campo dell'educazione si pratica la ricerca empirica in maniera insufficiente rispetto alle reali necessità (Vannini, 2012), e ciò comporta un abbassamento della qualità della stessa ricerca che, in questo modo, non riesce a sopperire ai bisogni né a finalizzare il suo intervento.

Nonostante il genuino intento di ricerca e il buon numero di studi sulla didattica della L2 che hanno avuto luogo negli ultimi anni (Balboni, 2012), si constata una mancanza di concretezza e spendibilità all'interno dell'impianto organizzativo delle scuole in Italia, poiché a fatica questi studi si sono inseriti nelle dinamiche decisionali.

I risultati del questionario riportano un forte desiderio, da parte della maggioranza del campione, di formazione e aggiornamento nella disciplina linguistica. Si tratta di un dato dalla valenza fortemente positiva, poiché comunica un livello decisamente alto di autocoscienza e di intenzione migliorativa. Di contro, l'espresso desiderio di aggiornamento sulle pratiche e sugli stili di insegnamento, potrebbe rivelare una volontà concettualmente sbagliata di pervenire al traguardo senza affrontare il percorso. La richiesta di proposte operative di modalità didattiche utili alla gestione di classi problematiche per la loro eterogeneità per scolarizzazione, livello linguistico, età e L 1 , si inserisce in questa linea di richiesta d'aiuto.

La soluzione al problema non è a portata di mano, ma non è nemmeno distante al punto da non essere raggiungibile.

Un primo passo potrebbe essere rappresentato dall'ampliamento dell'offerta di stage e tirocini presso gli istituti e le scuole eroganti corsi di italiano L2, che senz' altro agevolerebbe il percorso di introduzione e il confronto con i professionisti già operanti nel settore.

La formazione a distanza, per esempio, potrebbe aiutare nel superamento di difficoltà organizzative, mentre la messa a disposizione di risorse gratuite online accorcerebbe ulteriormente la distanza tra enti e persone (Cambiaghi, Milani, Pontani, 2005).

In aggiunta, la creazione di un' offerta formativa successiva al diploma, nella forma di corsi di laurea e master, costituirebbe il prerequisito necessario per la delineazione effettiva ed efficace della figura dell'insegnante di italiano L2 (Balboni, 2008).

L'operazione dovrebbe cominciare necessariamente, come si diceva, da un lavoro sinergico delle istituzioni coinvolte, con un'ottica moderna di più ampia accessibilità e con la costituzione di tavoli tecnici che creino proposte interdisciplinari in grado di far incontrare la teoria e la sua applicabilità (Vedovelli, 2002).

\section{Bibliografia}

BALBONI, P. E. (2008): «ltaliano L2: una via italiana». Studi di Glottodidattica, I, pp. 1731.

- (2012): Le sfide di Babele. Insegnare le lingue nelle società complesse. Novara: UTET. BERRUTO, G. (2004): Prima lezione di sociolinguistica. Roma; Bari: Laterza Editori. 
CAMBIAGHI, B., C. MILANI \& P. PONTANI, (a cura di) (2005): Europa plurilingue: Comunicazione e didattica. Milano: Vita e Pensiero.

DANESI, M. (2015): Il cervello in aula! Neurolinguistica e didattica per le lingue. Perugia: Guerra Edizioni.

DÖRNYEI, Z. (1998): "Motivation in second and foreign language learning". Language Teaching, 31 (3), pp. 117-135.

DUSI, P., B. GUIDETTI \& A. PORTERA (2015): L'educazione interculturale alla cittadinanza. Roma: Carocci editore.

EMANUEL, L. \& P. NITTI (2017): «Lifelong learning: aspetti pedagogici e didattici». Nuova Secondaria, 9, pp. 22-25.

FACCHETTI, G. (2007): Antropologia della scrittura. Milano: Arcipelago Edizioni.

FAVARO, G. (2014): A scuola nessuno è straniero. Firenze: Giunti.

FRABBONI, F. \& F. PINTO MINERVA (2005): Introduzione alla pedagogia generale. Roma: Editori Laterza.

MARINI, A. (2018): Manuale di neurolinguistica. Fondamenti teorici, tecniche di indagine, applicazioni. Roma: Carocci editore.

MEZZADRI, M. (2005): La qualità nell'insegnamento delle lingue straniere. Perugia: Guerra-Soleil.

NITTI, P. (2018): La didattica della lingua italiana per gruppi disomogenei. Brescia: Editrice La Scuola.

NUZZO, E. \& S. RASTELLI (201 1): Glottodidattica sperimentale. Nozioni, rappresentazioni e processing nell'apprendimento della seconda lingua. Roma: Carocci editore.

PEACOCK, M. (1997): "The effect of authentic materials on the motivation of EFL learners". ELT Journal, 51, pp. 144-56.

RASTELLI, S. (2009): Che cos'è la didattica acquisizionale. Roma: Carocci editore.

SANTALUCIA, D. (2015): "Competenza digitale e glottotecnologie per l'insegnante di italiano L2/LS). Italiano LinguaDue, 1, pp. 157-183.

SERRAGIOTTO, G. (2016): Nuove frontiere nella valutazione linguistica. Educazione Linguistica Language Education, 5(2), pp. 153-188.

VANNINI, I. (2012): Come cambia la cultura degli insegnanti. Metodi per la ricerca empirica in educazione. Milano: FrancoAngeli.

VEDOVELLI, M. (2002): L'italiano degli stranieri: storia, attualità e prospettive. Roma: Carocci editore. 